\title{
Characteristics of the structure in the Galactic polarized radio background at $350 \mathrm{MHz}$
}

\author{
M. Haverkorn ${ }^{1, \star}$, P. Katgert ${ }^{1}$, and A. G. de Bruyn ${ }^{2,3}$ \\ 1 Leiden Observatory, PO Box 9513, 2300 RA Leiden, The Netherlands \\ 2 ASTRON, PO Box 2, 7990 AA Dwingeloo, The Netherlands \\ e-mail: ger@astron.nl \\ 3 Kapteyn Institute, PO Box 800, 9700 AV Groningen, The Netherlands
}

Received 16 December 2002 / Accepted 21 March 2003

\begin{abstract}
Angular power spectra and structure functions of the Stokes parameters $Q$ and $U$ and polarized intensity $P$ are derived from three sets of radio polarimetric observations. Two of the observed fields have been studied at multiple frequencies, allowing determination of power spectra and structure functions of rotation measure $R M$ as well. The third field extends over a large part of the northern sky, so that the variation of the power spectra over Galactic latitude and longitude can be studied. The power spectra of $Q$ and $U$ are steeper than those of $P$, probably because a foreground Faraday screen creates extra structure in $Q$ and $U$, but not in $P$. The extra structure in $Q$ and $U$ occurs on large scales, and therefore causes a steeper spectrum. The derived slope of the power spectrum of $P$ is the multipole spectral index $\alpha_{P}$, and is consistent with earlier estimates. The multipole spectral index $\alpha_{P}$ decreases with Galactic latitude (i.e. the spectrum becomes flatter), but is consistent with a constant value over Galactic longitude. Power spectra of the rotation measure $R M$ show a spectral index $\alpha_{R M} \approx 1$, while the structure function of $R M$ is approximately flat. The structure function is flatter than earlier estimates from polarized extragalactic sources, which could be due to the fact that extragalactic source $R M$ probes the complete line of sight through the Galaxy, whereas as a result of depolarization diffuse radio polarization only probes the nearby ISM.
\end{abstract}

Key words. magnetic fields - polarization - techniques: polarimetric - ISM: magnetic fields - ISM: structure radio continuum: ISM

\section{Introduction}

The warm ionized gaseous component of the Galactic interstellar medium (ISM) shows structure in density and velocity on scales from AU to several kiloparsecs. The Galactic magnetic field is coupled to the motions of the ionized gas and has a comparable energy density, so that gas and magnetic field are in complex interaction. Detailed knowledge of the turbulent nature of the warm ISM and the structure in the Galactic magnetic field is essential for several fundamental studies of the Galaxy, including modeling of molecular clouds (e.g. Vázquez-Semadeni \& Passot 1999; Ostriker et al. 2001), heating of the ISM (Minter \& Balser 1997), star formation (e.g. Ferrière 2001), and cosmic ray propagation (Chevalier \& Fransson 1984).

Small-scale structure in the warm ISM and magnetic field can be well studied using radio polarimetric observations, of the synchrotron background in the Milky Way (e.g. Brouw \& Spoelstra 1976; Wieringa et al. 1993; Duncan et al. 1997, 1999;

Send offprint requests to: $\mathrm{M}$. Haverkorn, e-mail: mhaverkorn@cfa.harvard.edu

* Harvard-Smithsonian Center for Astrophysics, 60 Garden Street MS-67, Cambridge MA 02138, USA.
Uyanıker et al. 1999; Landecker et al. 2001; Gaensler et al. 2001), of pulsars (e.g. Rand \& Kulkarni 1989; Ohno \& Shibata 1993; Rand \& Lyne 1994; Han et al. 1999) or of polarized extragalactic point sources (e.g. Simard-Normandin \& Kronberg 1980; Clegg et al. 1992). At short wavelengths $(\lambda \lesssim 6 \mathrm{~cm})$, Faraday rotation is negligible, so that the measured polarization directly traces the magnetic field in the emitting region. At longer wavelengths, Faraday rotation measurements give additional information on density and magnetic field structure along the entire line of sight. Furthermore, depolarization processes define a distance beyond which polarized radiation is significantly depolarized, which depends on wavelength. So highfrequency measurements probe the total line of sight through the Galaxy, whereas low-frequency polarization observations only trace the nearby part of the ISM.

Specific intriguing small-scale structures and discrete objects have been studied in diffuse polarization observations (Gray et al. 1998; Haverkorn et al. 2000; Uyanıker \& Landecker 2002), but the spatial structures have also been analyzed statistically. Simonetti et al. (1984) and Simonetti \& Cordes (1986) studied the structure in Galactic rotation measure $R M$ by comparing $R M$ s of polarized extragalactic sources, and of separate components of the same source. The electron 
density seems to exhibit a power law structure function and therefore a power law angular spectrum (see also Armstrong et al. 1995; Minter \& Spangler 1996). Recently, statistical analysis of the diffuse Galactic polarized foreground have been pursued in the form of angular power spectrum studies (Tucci et al. 2000, 2002; Baccigalupi et al. 2001; Giardino et al. 2002; Bruscoli et al. 2002), with the objective of estimating the importance of the Galactic ISM as a foreground contaminator for CMBR polarization observations (e.g. Seljak 1997; Prunet et al. 2000).

In this paper, we study the statistical properties of the warm ISM and Galactic magnetic field by means of power spectra of Stokes parameters $Q$ and $U$, polarized intensity $P$ and rotation measure $R M$. We also derive the structure function of $R M$ to allow a comparison with earlier studies of $R M$ structure functions from polarized extragalactic sources. Furthermore, by careful selection of reliable $R M$ determinations (i.e. those with low $\chi^{2}$ of the linear fit to $\left.\phi\left(\lambda^{2}\right)\right)$ in the calculation of structure functions, we can obtain an estimate of how much the structure functions (and power spectra) are influenced by low-quality $R M \mathrm{~s}$.

We use data from three regions, all at positive Galactic latitudes, in which we observed the diffuse polarized emission at frequencies around $350 \mathrm{MHz}$ with the Westerbork Synthesis Radio Telescope (WSRT). The first two regions, each about 50 square degrees in size, in the constellations Auriga (Haverkorn et al. 2003a) and Horologium (Haverkorn et al. $2003 \mathrm{~b}$ ), are observed at multiple frequencies at a resolution of $\sim 5^{\prime}$. The third region is a part of the Westerbork Northern Sky Survey (WENSS, Rengelink et al. 1997), a high-resolution radio survey at $327 \mathrm{MHz}$ of the northern hemisphere (Schnitzeler et al., in prep). For those parts of the WENSS survey that were observed at night, polarization data are usable. Here we discuss polarization data from the region with $140^{\circ} \lesssim l \lesssim 170^{\circ}$ and $0^{\circ} \lesssim b \lesssim 30^{\circ}$.

In Sect. 2 we describe the three sets of data that we analyze in this paper. In Sect. 3 angular power spectrum analysis is introduced, power spectra of the data are presented and discussed and literature on the angular power spectra is briefly summarized. Section 4 gives structure functions for $R M$ in the two multi-frequency measurements. In Sect. 5 the results are discussed, and finally in Sect. 6 some conclusions are stated.

\section{The observations}

\subsection{Multi-frequency WSRT observations}

We carried out low frequency radio polarimetry with the Westerbork Synthesis Radio Telescope (WSRT), in two regions of the sky at positive latitudes, in the constellations of Auriga and Horologium. Data were obtained simultaneously at 8 frequencies around $350 \mathrm{MHz}$, each with a bandwidth of $5 \mathrm{MHz}$. Due to radio interference and hardware problems, only data in 5 frequency bands could be used, viz. those centered at the frequencies $341,349,355,360$, and $375 \mathrm{MHz}$. The multifrequency data allow the study of the frequency dependence of the polarization structure, and the determination of rotation measure $R M$. We use the technique of mosaicking (i.e. the telescopes cycle through a number of adjacent fields on the sky during a $12 \mathrm{hr}$ observation) to obtain a field of view that is larger than the primary beam. Mosaicking also suppresses instrumental polarization to below 1\% (see Haverkorn 2002). Maps of the Stokes parameters $I, Q$, and $U$ were derived from the observed visibilities. $R M \mathrm{~s}$ were computed straightforwardly from the linear relation between polarization angle $\phi$ and $\lambda^{2}$. Because the observed $R M$ values are small $(|R M| \lesssim$ $10 \mathrm{rad} \mathrm{m}^{-2}$ ), there is no $n 180^{\circ}$ ambiguity in $\phi$, and $R M \mathrm{~s}$ can be computed with $\left|\phi\left(\lambda_{i}\right)-\phi\left(\lambda_{j}\right)\right|<90^{\circ}$ for adjacent wavelengths $\lambda_{i}$ and $\lambda_{j}$. We define a determination of $R M$ in a particular beam reliable if (1) the reduced $\chi^{2}$ of the linear $\phi\left(\lambda^{2}\right)$-relation $\chi_{\text {red }}^{2}<2$, and (2) the polarized intensity averaged over wavelength $\langle P\rangle>20 \mathrm{mJy} /$ beam (i.e. $\sim 4 \sigma)$.

The maximum baseline of the observations was $2700 \mathrm{~m}$, yielding a resolution of $1^{\prime}$, but smoothing of the Stokes $Q$ and $U$ data (using a Gaussian taper in the $(u, v)$-plane) was applied to obtain a better signal-to-noise. The taper has a value of 0.25 at a baseline value around $300 \mathrm{~m}$, where the exact values were chosen so that the beam size is identical at all 5 frequencies, viz. $5.0^{\prime} \times 5.0^{\prime} \operatorname{cosec} \delta$.

The first region, in the constellation Auriga, is centered on $(l, b)=\left(161^{\circ}, 16^{\circ}\right)$ and is about $9^{\circ} \times 7^{\circ}$ in size. The left panel in Fig. 1 shows the polarized intensity $P$ at $349 \mathrm{MHz}$ in the Auriga region at $5.0^{\prime}$ resolution. We do not show the original $1^{\prime}$ resolution map, because it is noise-dominated. The maximum polarized brightness temperature in the map is $T_{\mathrm{b}, \mathrm{pol}} \approx 13 \mathrm{~K}$, and the noise is about $0.45 \mathrm{~K}$. Rotation measures in the Auriga region are shown in the right panel of Fig. 1 as circles superimposed on the grey scale map of $P$ at $349 \mathrm{MHz}$. The diameter of each circle indicates the value of the $R M$ at that position, where filled circles denote positive $R M \mathrm{~s}$, and open circles negative $R M \mathrm{~s}$. We show only the $R M \mathrm{~s}$ that are reliably determined, and only one in four independent beams.

The region in the constellation Horologium, centered at $(l, b)=\left(137^{\circ}, 7^{\circ}\right)$, was observed in the same way as the Auriga region, at the same five frequencies, and with the same taper applied. Figure 2 shows the polarized intensity $P$ at $349 \mathrm{MHz}$ in the left panel, and $R M \mathrm{~s}$ in the form of overlaid circles in the right panel. $R M \mathrm{~s}$ are coded in the same way as in Fig. 1 . The maximum polarized brightness temperature is $T_{\mathrm{b}, \mathrm{pol}} \approx 17 \mathrm{~K}$, and the noise is a little higher than that in the Auriga field, about $0.65 \mathrm{~K}$.

The polarized intensity is not corrected for noise bias $P_{\text {debias }}=\sqrt{\left(Q_{\mathrm{obs}}^{2}+U_{\mathrm{obs}}^{2}\right)-\sigma^{2}}$ (for $\left.P>\sigma\right)$. However, $P$ generally has a $S / N>4-5$, for which the debiasing does not alter the data by more than $2-3 \%$. Furthermore, power spectra are not affected by debiasing except at zero frequency.

In both fields there is no small-scale structure visible in the map of total intensity Stokes $I$, despite the ubiquitous structure on arcminute and degree scales in $P$. Because the large-scale $\left(\gtrsim 1^{\circ}\right)$ component of $I$ cannot be measured with the WSRT due to missing short spacings, we used the Haslam et al. (1981, 1982) radio survey at $408 \mathrm{MHz}$ to estimate the total intensity $I$ to be $\sim 34 \mathrm{~K}$ and $\sim 47 \mathrm{~K}$ in Auriga and Horologium, respectively. The polarized intensity $P$ shows structure up to $\sim 10-15 \mathrm{~K}$ on scales from arcminutes to degrees. Due to the 

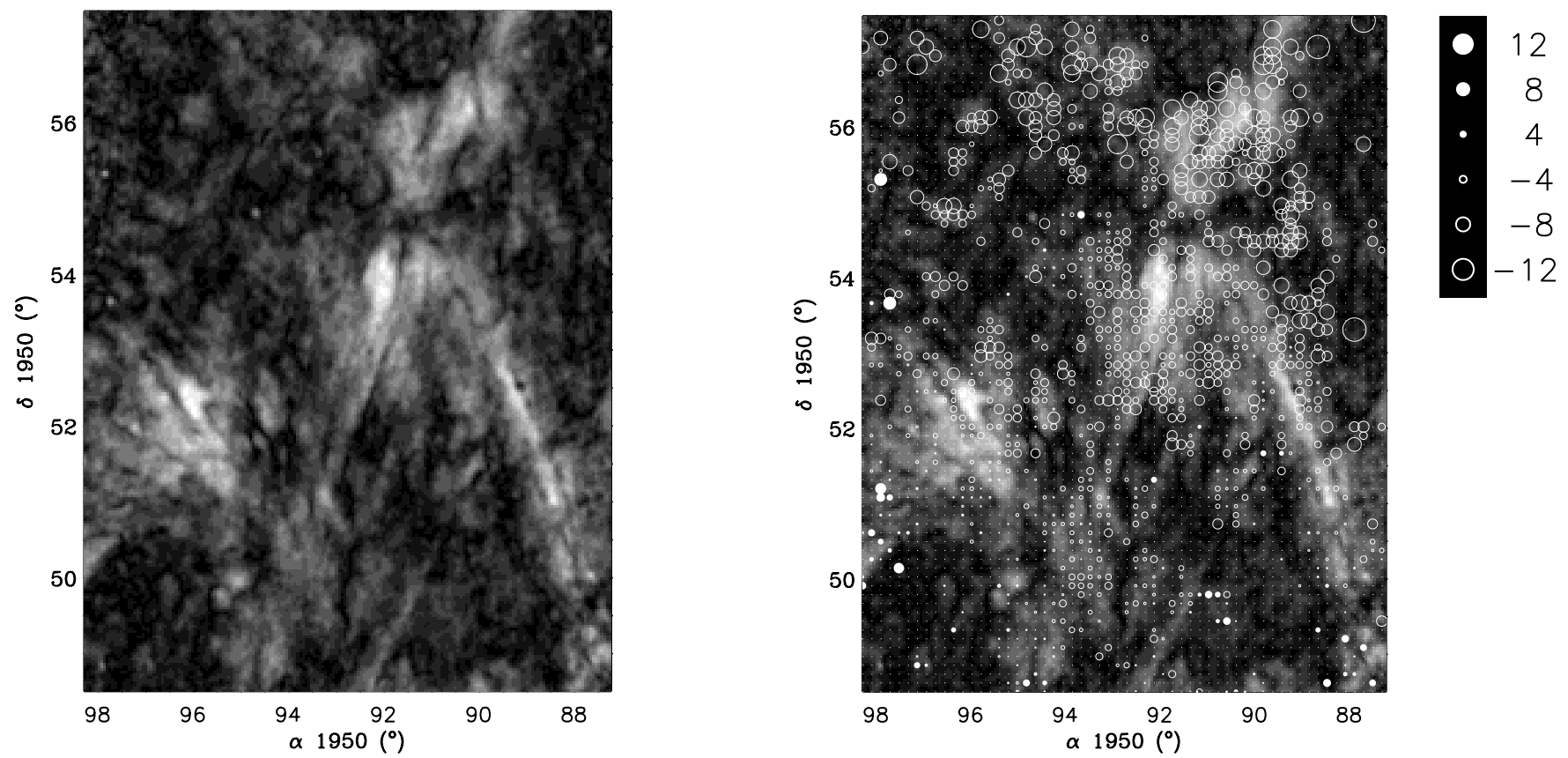

Fig. 1. Grey scale representation of the polarized intensity $P$ at $349 \mathrm{MHz}$ and rotation measure $R M$, of the region in the constellation of Auriga centered at $(l, b)=\left(161^{\circ}, 16^{\circ}\right)$ at a resolution of $\sim 5^{\prime}$. The grey scale is saturated at $P=95 \mathrm{mJy} / \mathrm{beam}$ (white) $\left(T_{\mathrm{b}, \text { pol }}=12 \mathrm{~K}\right)$. The right panel shows $R M$ in superimposed circles, where the diameters of the circles indicate $R M$, and filled circles denote positive $R M \mathrm{~s}$. The scaling of $R M$ on the right-hand side is in $\mathrm{rad}^{-2}$.
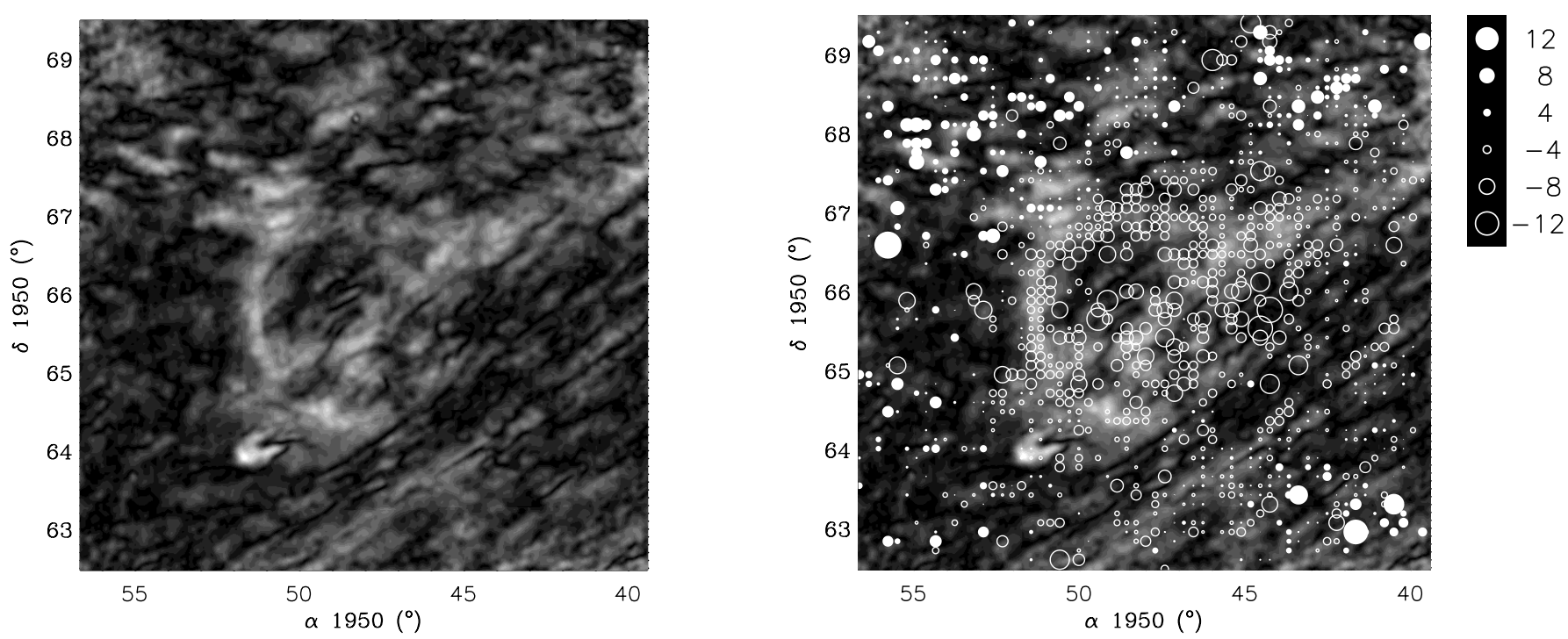

Fig. 2. $P$ and $R M$ for the region in the constellation of Horologium centered at $(l, b)=\left(137^{\circ}, 7^{\circ}\right)$. Notation as in Fig. 1 , where the maximum $P=95 \mathrm{mJy} /$ beam corresponds to $T_{\mathrm{b}, \mathrm{pol}}=13.9 \mathrm{~K}$.

lack of corresponding structure in $I$, the structure in polarized intensity cannot be due to small-scale synchrotron emission but must be due to other, instrumental and depolarization, mechanisms.

In a medium that emits synchrotron radiation and simultaneously causes Faraday rotation, the polarized emission is depolarized by so-called depth depolarization, due to the vector averaging of contributions from different parts of the line of sight. This, together with beam depolarization (due to angle structure within one synthesized beam), produces structure in $P$. Furthermore, the insensitivity of the interferometer to large-scale structure can cause additional structure in $P$. However, we have shown that this effect cannot be very important in these observations (Haverkorn et al. 2003c).

\subsection{Polarization data from the WENSS survey}

The Westerbork Northern Sky Survey (WENSS, Rengelink et al. 1997) is a low-frequency radio survey that covers the whole sky north of $\delta=30^{\circ}$ at $325 \mathrm{MHz}$ to a limiting flux 


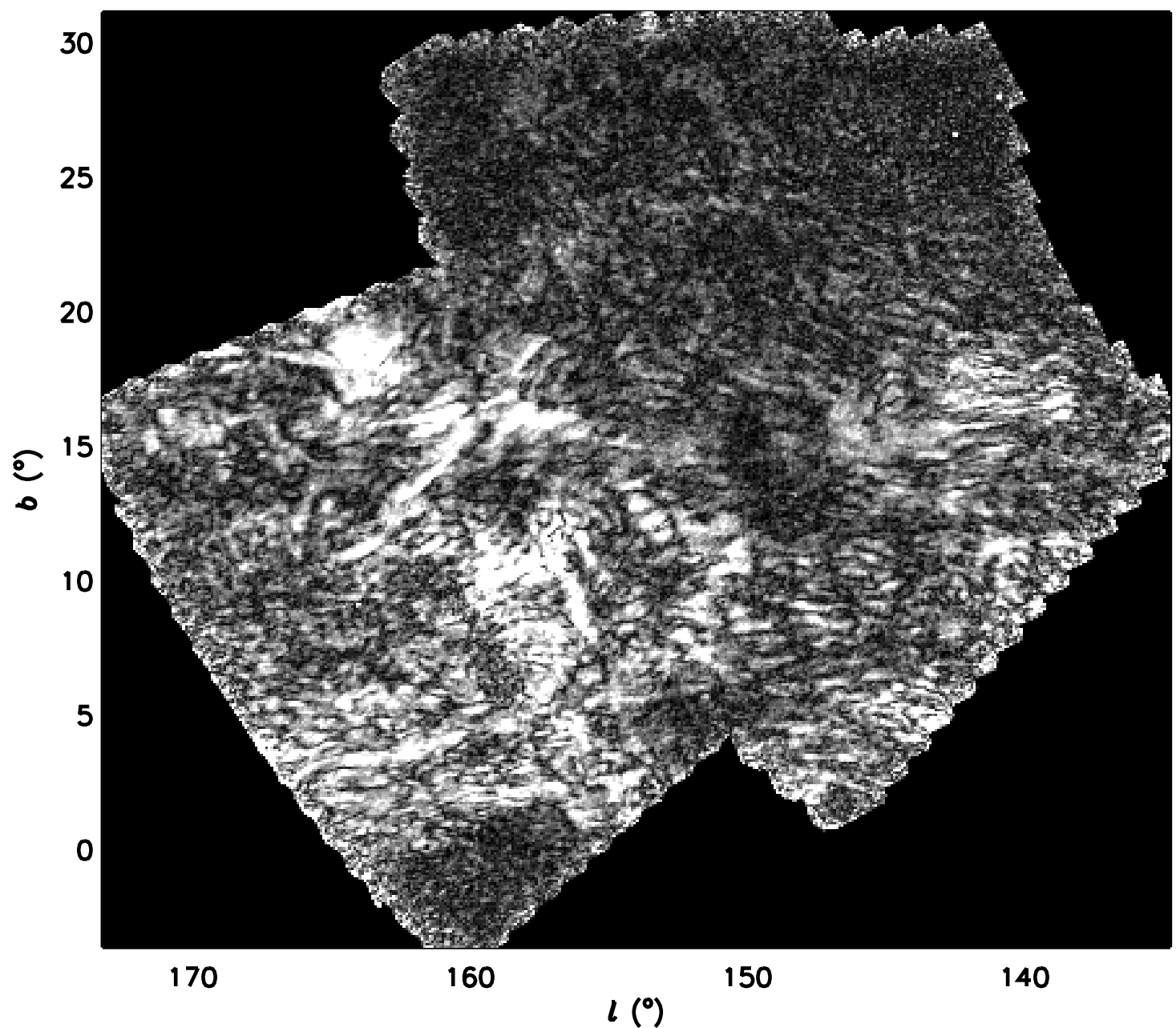

Fig. 3. Grey scale representation of the polarized intensity of the polarization part of the WENSS survey at $325 \mathrm{MHz}$, where white denotes a maximum intensity of $35 \mathrm{mJy} /$ beam $\left(T_{\mathrm{b}, \mathrm{pol}} \approx 17 \mathrm{~K}\right.$ to $25 \mathrm{~K}$, depending on declination). The data were smoothed with a $500 \mathrm{~m}$ taper, and have a resolution of $\sim 2.5^{\prime}$.

density of approximately $18 \mathrm{mJy}(5 \sigma)$ and with a resolution of $54^{\prime \prime} \times 54^{\prime \prime} \operatorname{cosec} \delta$

Polarization data taken during the day are greatly affected by solar radiation, which is detected in sidelobes. Furthermore, ionospheric Faraday rotation rapidly changes during sunrise and sunset, causing a reduction of the apparent polarized intensity, and adding much noise. However, in mosaics observed (almost) entirely during night time, the polarization data are of good quality. As a result, we could make a "supermosaic" of a large region of $\sim 30^{\circ} \times 35^{\circ}$, which we will refer to as the WENSS polarization region. Details on the observations and correction for ionospheric Faraday rotation are given in Schnitzeler et al. (in prep).

Figure 3 shows polarized intensity $P$ in the WENSS polarization region, resampled in Galactic longitude and latitude. For this analysis, a Gaussian taper with value 0.25 at a baseline of $500 \mathrm{~m}$ was applied, which yields a resolution of $\sim 2.5^{\prime}$. In the figure, $P$ saturates at $35 \mathrm{mJy} / \mathrm{beam}$, which coincides with a polarized brightness temperature of $\sim 17 \mathrm{~K}$ to $\sim 25 \mathrm{~K}$, depending on declination. The average $P \approx 2.6 \mathrm{mJy} /$ beam. Note that these observations are taken in a single $5 \mathrm{MHz}$ wide frequency band, so rotation measure data are not available.

\section{Angular power spectrum analysis}

\subsection{Determination of the multipole spectral index}

To quantify the structure in the polarization maps, we calculate angular power spectra $P S(\ell)$ as a function of multipole $\ell$. A multipole $\ell$ is a measure of angular scales equivalent to wave number, and is defined as $\ell \approx 180^{\circ} / \theta$, where $\theta$ is the angular scale in degrees. The angular power spectrum $P S$ of a radiation field $X$ is the square of the Fourier transform of $X$ : $P S_{X}(\ell)=|\mathcal{F}(X)|^{2}$, where $\mathcal{F}$ denotes the Fourier transform. Here, the observable $X$ can be either Stokes $Q$, Stokes $U$, polarized intensity $P$ or rotation measure $R M$. The power spectra were computed in two dimensions, and averaged over azimuth in radial bins. The multipole spectral index $\alpha$, defined as $P S_{X}(\ell) \propto \ell^{-\alpha}$, is calculated from a log-log fit to the power spectrum. In the tapered data, multipoles with higher values of $\ell$ are affected by the tapering, and in the untapered data higher multipoles are dominated by noise. Multipoles with $\ell \lesssim 200$ correspond to angular scales $\theta \gtrsim 1^{\circ}$, to which the WSRT is not sensitive.

The visibilities from which a map is made are $V_{\text {map }}(u, v)=$ $V_{\text {obs }}(u, v) T(u, v)$, where $V_{\text {obs }}$ are the observed visibilities, and 


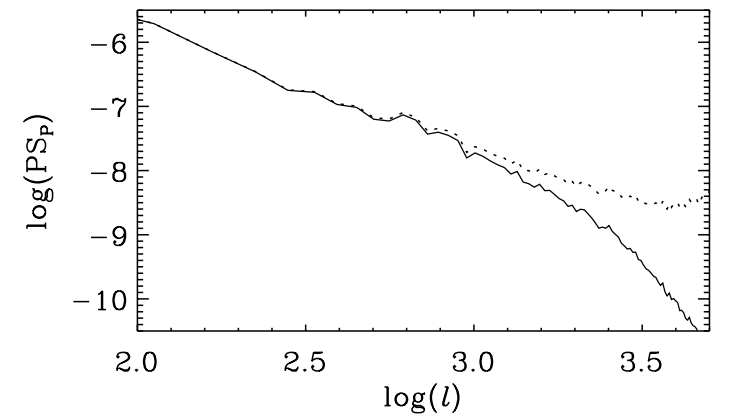

Fig. 4. Power spectrum of $P$ at $341 \mathrm{MHz}$ in the Auriga field. The solid line shows the observed data, the dotted line is corrected for the $300 \mathrm{~m}$ taper.

$T(u, v)$ is the taper function. The calculated intensity of the tapered data is $\mathcal{F}\left(V_{\text {map }}\right)=\mathcal{F}\left(V_{\text {obs }}\right) * \mathcal{F}(T)$, where $\mathcal{F}$ is a Fourier transform and the asterisk denotes convolution. The power spectrum of the Stokes parameter $X, P S(X)$, is then:

$P S_{X}(\ell)=|\mathcal{F}(X)|^{2}=\left|\mathcal{F}\left(X_{\mathrm{obs}}\right)\right|^{2} T^{2}=P S_{X, \mathrm{obs}}(\ell) T^{2}$

where $X$ is Stokes $Q$ or $U$. Although polarized intensity $P$ is derived from $Q$ and $U$ and thus not directly observed, correction for the taper in the same way as for $Q$ and $U$ power spectra is a good approximation. As an illustration, Fig. 4 shows the power spectrum of $P$ of the tapered data in the Auriga region at $341 \mathrm{MHz}$ (solid line). The dotted line is the same spectrum, but corrected for the tapering according to Eq. (1). The power law behavior extends to $\log (\ell) \approx 3.6$.

\subsection{Power spectra from the multi-frequency WSRT studies}

In Fig. 5, we show the power spectra of $P$ in the Auriga and Horologium regions at 5 frequencies, both for the tapered (upper curve) and untapered data (lower curve) in the same plot. The amplitudes of the power spectra of the tapered data are lower than those of the untapered data only because the intensities are expressed in mJy/beam. Because the beam widths are different for the two datasets, this gives a difference in the magnitude of $P$ in tapered and untapered data. The power spectra of $R M$ in the Auriga and Horologium field are given in Fig. 6. Only tapered data give reliable enough $R M$ determinations to produce power spectra for them.

Figures 7 and 8 show power spectra for the Stokes parameters $Q$ and $U$ in the Auriga and Horologium region respectively, again for tapered and untapered data. The corresponding multipole spectral indices $\alpha$, derived for a multipole range of $400<\ell<1500$, are given in Table 1 .

At small scales (large $\ell$ ), the power spectra of the untapered data flatten out due to the noise in the maps, while the low-resolution data steepen due to the tapering, as illustrated in Fig. 4. At large scales, the $Q$ and $U$ power spectra of the tapered data show a decrease. This decrease could be due to the lack of large-scale structure (see Sect. 2.1), but then it is hard to explain why there is no such decline in $P$.

The power spectra of $Q$ and $U$ are steeper and have a larger amplitude than the power spectra of $P$. This could be caused
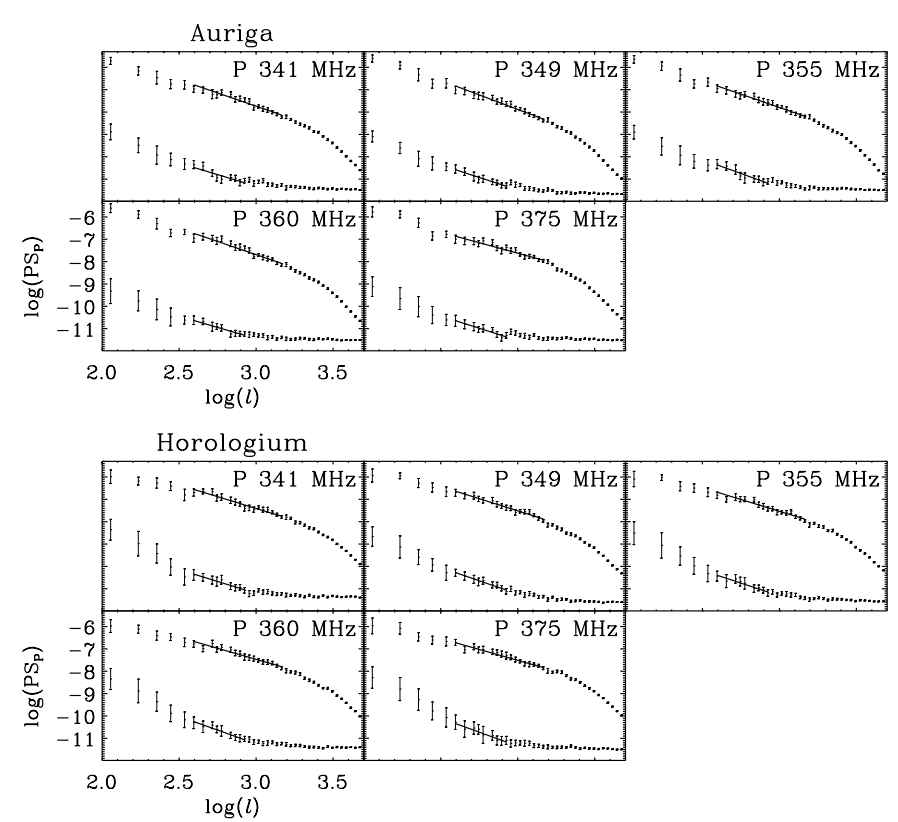

Fig. 5. Power spectra of polarized intensity $P$ for 5 frequency bands in the Auriga region (top) and Horologium region (bottom). In each plot, the upper line of symbols denotes the tapered data, the lower line the untapered data, and the solid lines are linear fits to the spectra. In the untapered data, the spectrum is flattened at high $\ell$ due to noise.
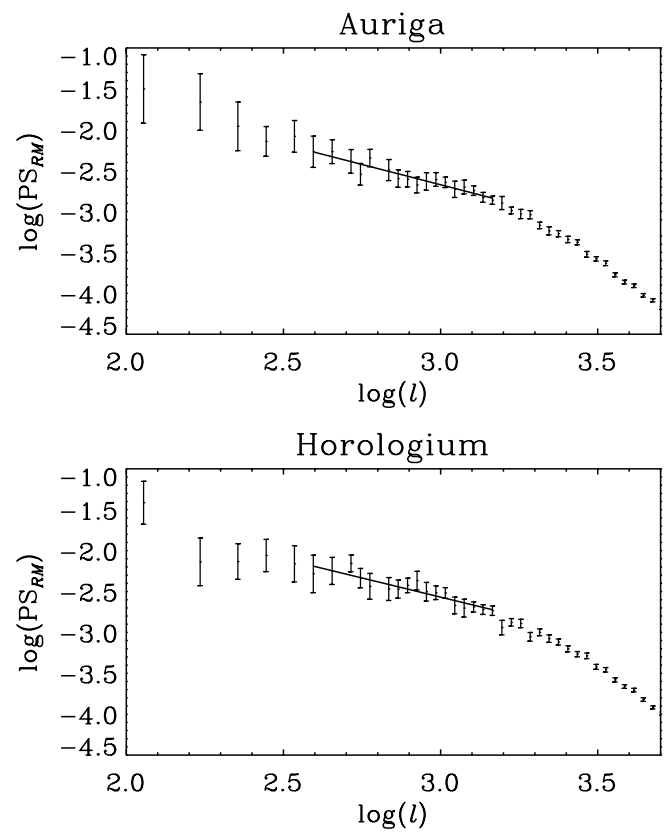

Fig. 6. Power spectra of $R M$ in the Auriga region (top) and Horologium region (bottom), derived from the tapered data.

by the presence of a Faraday screen in front of the emitting region. A Faraday screen will rotate the polarization angle, and so induce extra structure in $Q$ and $U$, while leaving $P$ unaltered. This results in a higher amplitude of the power spectrum. As the Faraday screen consists of foreground material, its angular size is large, steepening the spectrum. This effect was also noticed by Tucci et al. (2002).

The logarithmic slope of the power spectrum of polarized intensity is $\alpha_{P} \approx 2.1-2.3$ (Table 1 ), which is slightly higher 
Table 1. Multipole spectral indices $\alpha$ for observed polarized intensity $P$, Stokes $Q$ and Stokes $U$, for 5 frequencies and their average over frequency, and $\alpha_{R M}$, in the Auriga and Horologium regions. Values for $\alpha_{P}, \alpha_{Q}$ and $\alpha_{U}$ are given for tapered data, denoted by a subscript " $t$ ", and untapered data. The multipole ranges used to derive $\alpha$ were $400<\ell<1500$. Only for $\alpha_{P}$ of the untapered data, the range was smaller because of flattening of the spectrum at higher $\ell$.

Auriga

\begin{tabular}{lcccccc}
\hline \hline & $341 \mathrm{MHz}$ & $349 \mathrm{MHz}$ & $355 \mathrm{MHz}$ & $360 \mathrm{MHz}$ & $375 \mathrm{MHz}$ & mean \\
\hline$\alpha_{P, t}$ & $2.37 \pm 0.19$ & $2.59 \pm 0.20$ & $2.38 \pm 0.19$ & $2.35 \pm 0.17$ & $1.88 \pm 0.18$ & $2.32 \pm 0.08$ \\
$\alpha_{P}$ & $2.06 \pm 0.54$ & $2.23 \pm 0.46$ & $2.49 \pm 0.60$ & $2.00 \pm 0.47$ & $2.20 \pm 0.59$ & $2.20 \pm 0.24$ \\
$\alpha_{Q, t}$ & $3.55 \pm 0.20$ & $3.72 \pm 0.21$ & $3.59 \pm 0.20$ & $3.43 \pm 0.19$ & $2.50 \pm 0.20$ & $3.36 \pm 0.09$ \\
$\alpha_{Q}$ & $3.13 \pm 0.24$ & $3.16 \pm 0.25$ & $3.16 \pm 0.24$ & $3.00 \pm 0.24$ & $3.12 \pm 0.25$ & $3.12 \pm 0.11$ \\
$\alpha_{U, t}$ & $3.69 \pm 0.19$ & $3.89 \pm 0.21$ & $3.79 \pm 0.21$ & $3.69 \pm 0.21$ & $3.49 \pm 0.20$ & $3.71 \pm 0.09$ \\
$\alpha_{U}$ & $3.25 \pm 0.24$ & $3.20 \pm 0.25$ & $3.23 \pm 0.24$ & $3.13 \pm 0.24$ & $3.18 \pm 0.53$ & $3.20 \pm 0.11$ \\
$\alpha_{R M, t}$ & & & & & & $0.99 \pm 0.08$ \\
\hline
\end{tabular}

\section{Horologium}

\begin{tabular}{lcccccc}
\hline \hline & $341 \mathrm{MHz}$ & $349 \mathrm{MHz}$ & $355 \mathrm{MHz}$ & $360 \mathrm{MHz}$ & $375 \mathrm{MHz}$ & mean \\
\hline$\alpha_{P, t}$ & $2.11 \pm 0.19$ & $2.18 \pm 0.19$ & $2.11 \pm 0.18$ & $1.98 \pm 0.19$ & $1.95 \pm 0.19$ & $2.07 \pm 0.08$ \\
$\alpha_{P}$ & $2.12 \pm 0.63$ & $2.36 \pm 0.55$ & $2.26 \pm 0.66$ & $2.47 \pm 0.68$ & $2.49 \pm 0.91$ & $2.34 \pm 0.31$ \\
$\alpha_{Q, t}$ & $2.58 \pm 0.22$ & $3.17 \pm 0.19$ & $2.96 \pm 0.19$ & $2.90 \pm 0.20$ & $2.78 \pm 0.20$ & $2.89 \pm 0.09$ \\
$\alpha_{Q}$ & $2.15 \pm 0.26$ & $2.92 \pm 0.22$ & $2.57 \pm 0.23$ & $2.60 \pm 0.23$ & $2.32 \pm 0.25$ & $2.51 \pm 0.11$ \\
$\alpha_{U, t}$ & $2.56 \pm 0.22$ & $3.14 \pm 0.20$ & $3.04 \pm 0.19$ & $3.22 \pm 0.20$ & $3.24 \pm 0.17$ & $3.04 \pm 0.09$ \\
$\alpha_{U}$ & $2.07 \pm 0.26$ & $3.00 \pm 0.22$ & $2.69 \pm 0.23$ & $2.85 \pm 0.23$ & $2.65 \pm 0.25$ & $2.65 \pm 0.11$ \\
$\alpha_{R M, t}$ & & & & & & $0.94 \pm 0.10$ \\
\hline
\end{tabular}
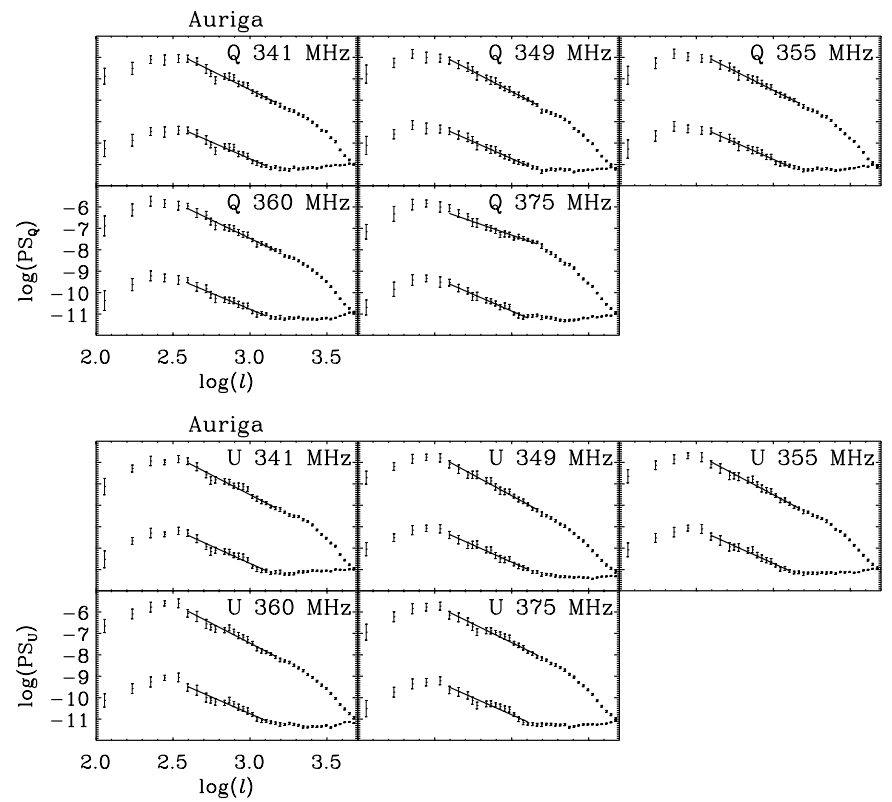

Fig. 7. Power spectra of Stokes $Q$ (top) and Stokes $U$ (bottom) for 5 frequencies in the Auriga region. Notation as in Fig. 5.

than most earlier estimates from the literature (although those are taken at higher frequencies, see Sect. 3.4). However, note that the Auriga and Horologium regions were selected for their conspicuous structure in $P$, so we expect these regions to show more structure on large (degree) scales than the "average" ISM, and thus exhibit a steeper spectrum. The power spectra of $Q$ and $U$ in the Auriga region are somewhat steeper than in Horologium, indicating that the Horologium region probably contains more small-scale structure in the Faraday screen than the Auriga region.
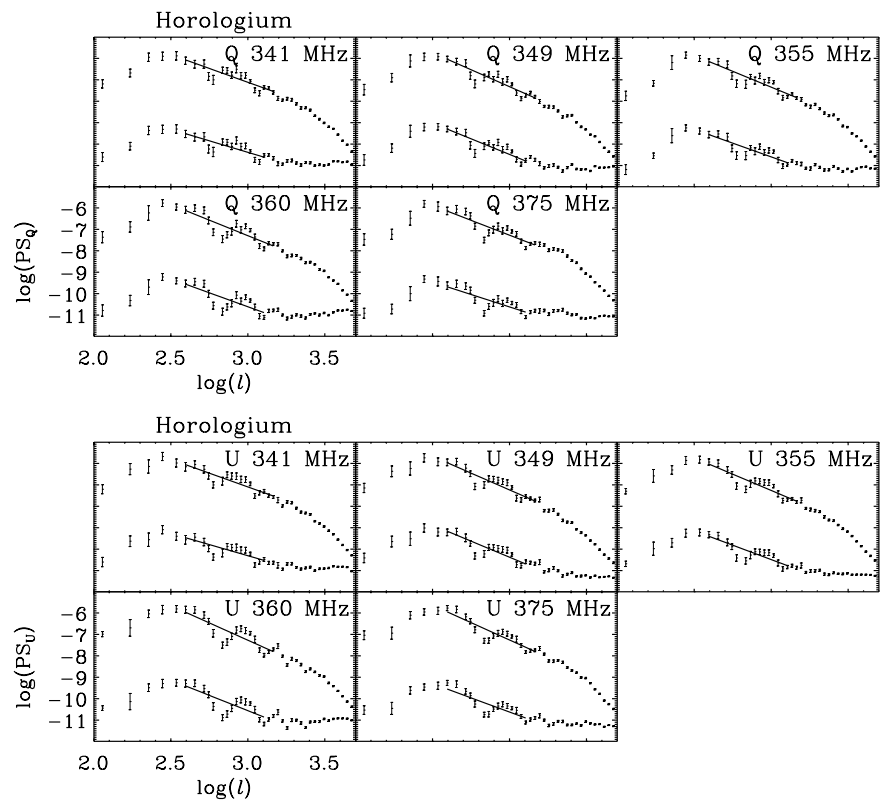

Fig. 8. Power spectra of Stokes $Q$ (top) and Stokes $U$ (bottom) for 5 frequencies in the Horologium region. Notation as in Fig. 5.

The power spectra of $R M$ are shallower than the $Q, U$ or $P$ power spectra. In fact, we do not expect a direct correspondence between the multipole spectral indices of $R M$ and $P$ (or $Q, U)$, as the former describes very directly the electron content and magnetic field in the ISM (integrated over the line of sight), whereas in the latter case the polarized radiation is modulated by Faraday rotation and depolarization. 
Table 2. Multipole spectral indices $\alpha$ for observed polarized intensity $P$, Stokes $Q$ and Stokes $U$ in 11 subfields in the WENSS polarization region at $325 \mathrm{MHz}$.

\begin{tabular}{lcccc}
\hline \hline No. & $(l, b)\left({ }^{\circ},{ }^{\circ}\right)$ & $\alpha_{P}$ & $\alpha_{Q}$ & $\alpha_{U}$ \\
\hline 1 & $(159,04)$ & $1.67 \pm 0.08$ & $2.55 \pm 0.09$ & $2.43 \pm 0.10$ \\
2 & $(165,11)$ & $1.48 \pm 0.08$ & $2.10 \pm 0.08$ & $2.06 \pm 0.08$ \\
3 & $(158,11)$ & $1.84 \pm 0.09$ & $2.50 \pm 0.09$ & $2.20 \pm 0.09$ \\
4 & $(151,11)$ & $1.70 \pm 0.08$ & $2.09 \pm 0.09$ & $2.02 \pm 0.09$ \\
5 & $(144,11)$ & $1.63 \pm 0.08$ & $2.28 \pm 0.10$ & $2.31 \pm 0.10$ \\
6 & $(143,18)$ & $1.24 \pm 0.09$ & $1.96 \pm 0.10$ & $2.04 \pm 0.10$ \\
7 & $(150,18)$ & $0.99 \pm 0.08$ & $1.44 \pm 0.08$ & $1.47 \pm 0.09$ \\
8 & $(157,18)$ & $1.67 \pm 0.09$ & $2.33 \pm 0.09$ & $2.33 \pm 0.09$ \\
9 & $(157,25)$ & $0.73 \pm 0.08$ & $1.24 \pm 0.08$ & $1.18 \pm 0.09$ \\
10 & $(150,25)$ & $0.90 \pm 0.08$ & $1.18 \pm 0.08$ & $1.44 \pm 0.08$ \\
11 & $(144,25)$ & $0.68 \pm 0.08$ & $0.77 \pm 0.08$ & $0.63 \pm 0.08$ \\
\hline
\end{tabular}

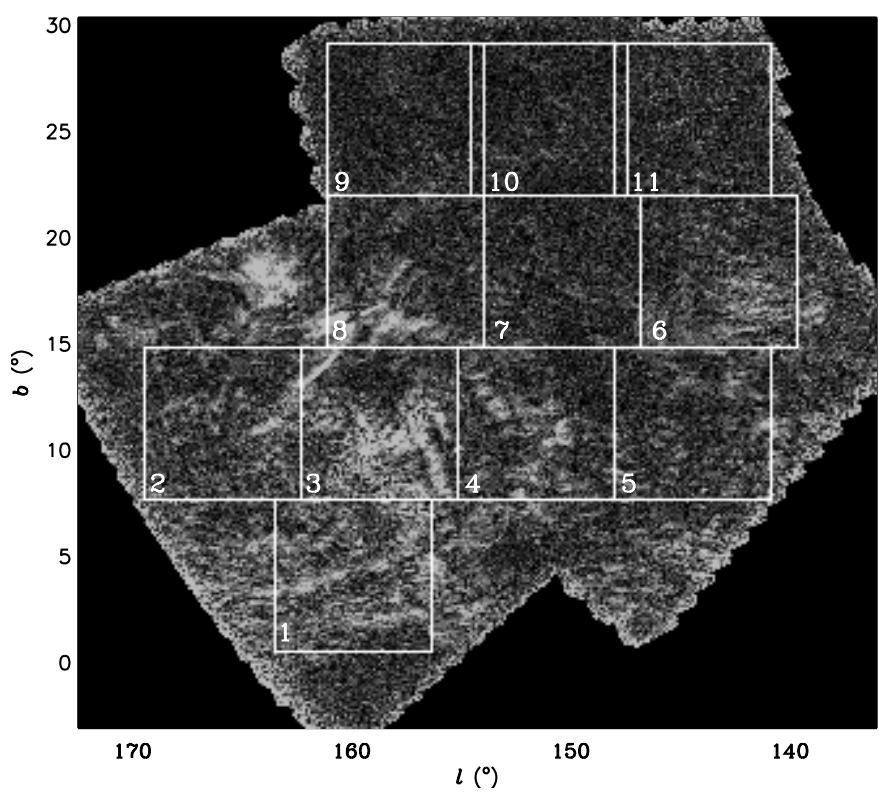

Fig. 9. Polarized intensity at $325 \mathrm{MHz}$ of the WENSS region in grey scale, superimposed with white boxes denoting the numbered subfields over which angular power spectra were computed.

\subsection{Power spectra from the WENSS polarization region}

In the WENSS polarization region, power spectra were evaluated for subfields, to study possible dependences of the multipole spectral index on Galactic longitude and/or latitude. The 11 subfields are shown in Fig. 9, superimposed on grey scale maps of $P$. The power spectra of polarized intensity $P$ are shown in Fig. 10, where the subfields are arranged as in Fig. 9. The power spectra in subfields 9, 10 and 11, at high Galactic latitude $b$, have a lower amplitude than the power spectra at lower $b$, which is consistent with the decreasing amount of $P$ at higher $b$ visible in Fig. 3 .

The multipole spectral indices of the power spectra of $P$, $Q$ and $U$ are given in Table 2, and the dependence of $\alpha_{P}$ on Galactic longitude and latitude is shown in Fig. 11. The

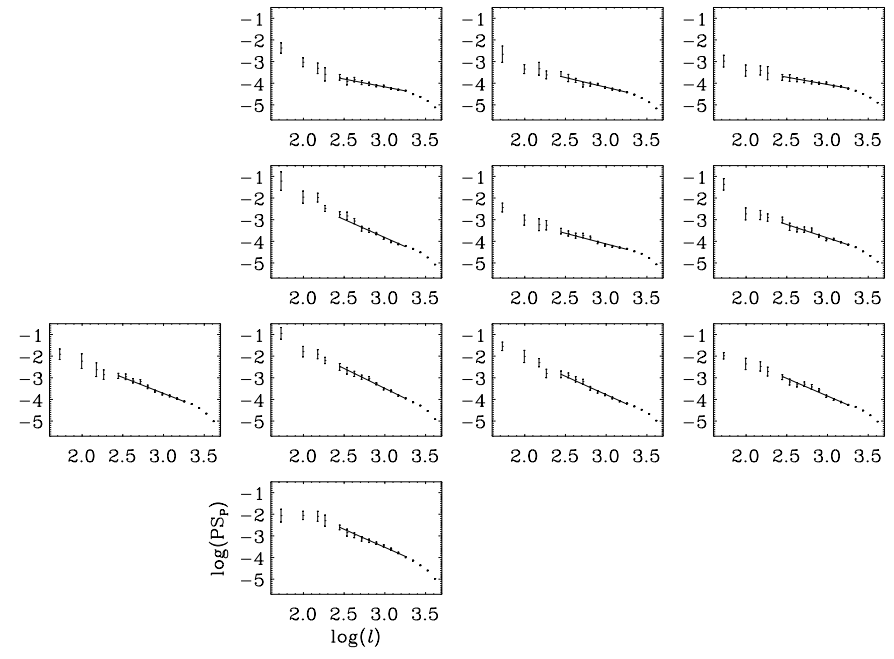

Fig. 10. Power spectra of polarized intensity $P$ in subfields in the WENSS polarization region. The plots are arranged as in Fig. 9.
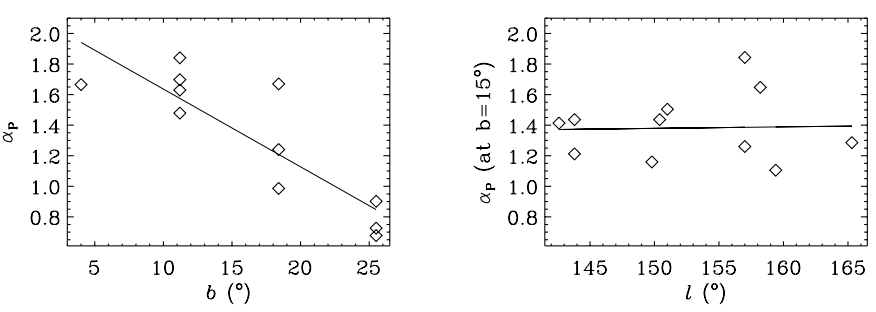

Fig. 11. Dependence of multipole spectral index $\alpha_{P}$ on Galactic latitude $b$ (left) and longitude $l$ (right), for 11 subfields in the polarization part of the WENSS survey. The spectral indices in the right plot are normalized to the latitude gradient shown in the left plot.

observed decrease of spectral index with increasing latitude (i.e. power spectra become flatter with increasing latitude) indicates a decrease in the amount of large-scale structure with increasing latitude. The dependence of spectral index on longitude is computed after rescaling of the data to a standard latitude of $b=15^{\circ}$. The fitted slopes in Fig. 11, and slopes determined for $\alpha_{Q}$ and $\alpha_{U}$ in a similar way, are:

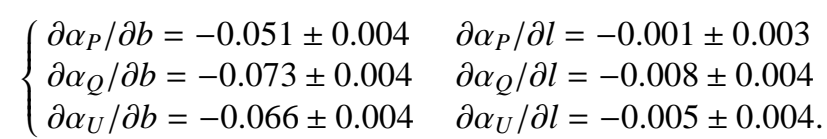

In summary, the spectral index $\alpha$ in $P, Q$ and $U$ decreases with increasing Galactic latitude, and is consistent with no dependence of $\alpha$ on Galactic longitude. Although the small errors in the derived slopes suggest a good determination of the slope, the large spread of the data points in Fig. 11 indicates that a linear gradient is not the perfect model to describe the data.

The decrease of $\alpha$ with latitude indicates that there is more structure in polarization on larger scales at lower latitude. Thus, although small-scale structure in polarization is seen up to very high latitudes, even at frequencies as low as $350 \mathrm{MHz}$ (Katgert \& de Bruyn 1999), the amount of structure and its spectral index decrease with latitude, at least in this region of the sky. The region studied here may be special, as it is situated at the edge of a region of high polarization (the "fan region", see e.g. Brouw \& Spoelstra 1976), which is thought to have little 

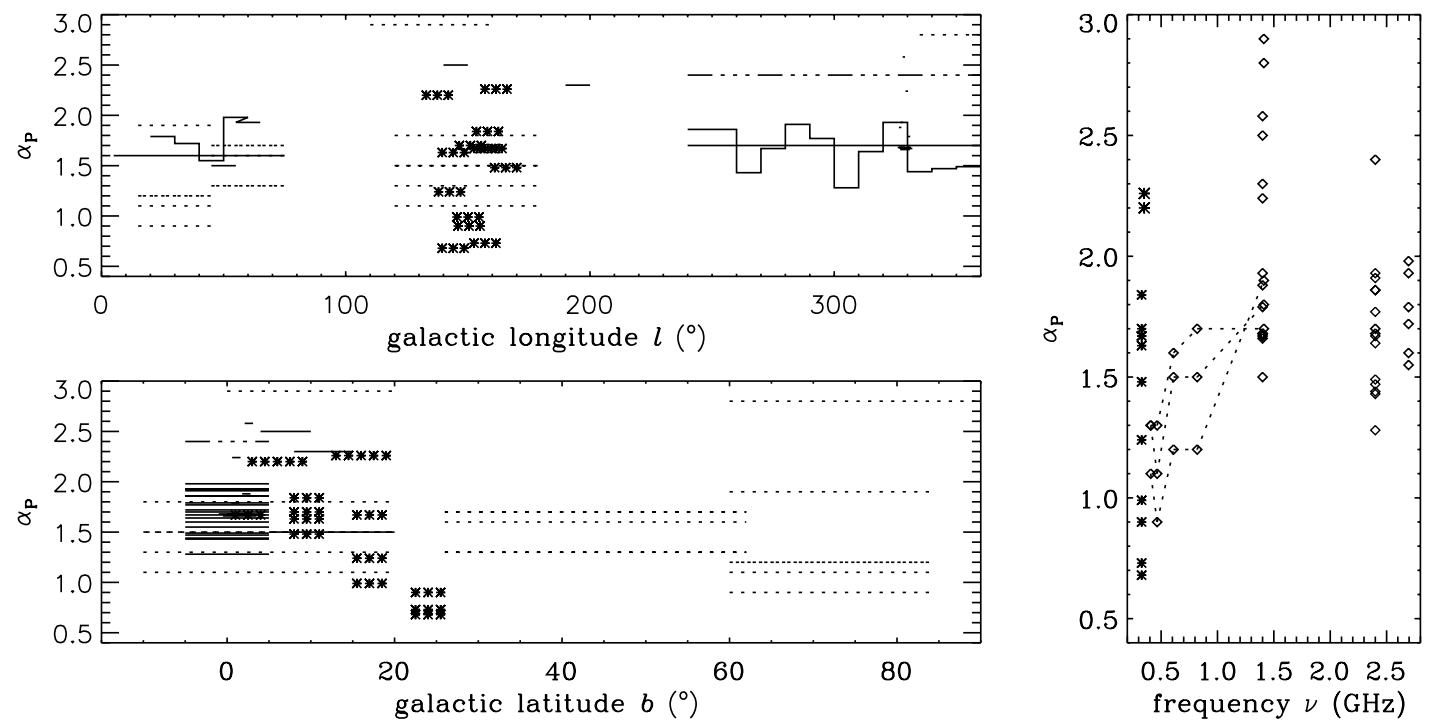

Fig. 12. Estimates of multipole spectral indices $\alpha_{P}(\ell)$ from our observations and from the literature, as a function of Galactic longitude (top), Galactic latitude (bottom), and frequency (right). In the left-hand plots, solid lines denote high multipole numbers $(100<\ell<6000)$, the dashed-dotted line is an intermediate multipole range $(30<\ell<200)$ and the dotted lines give small multipoles $(10<\ell<80)$. In the right-hand (frequency) plot, the dotted lines connect observations of the same region made at different frequencies. The WSRT observations discussed in this paper are shown by asterisks.

structure in magnetic field and/or electron density compared to its surroundings. This high-polarization region extends to $b \approx 20^{\circ}$, and its edge could be responsible for the decrease in structure on scales of $\sim 1^{\circ}$.

The spectral indices in the Auriga $\left(b=16^{\circ}\right)$ and Horologium $\left(b=7^{\circ}\right)$ regions are higher than implied by Fig. 11. This might be due to the way in which the Auriga and Horologium regions were selected, viz. because of their remarkable structure on degree scales.

\subsection{Existing literature of power spectra from diffuse polarization}

Much work has been done on the determination of power spectra of the diffuse Galactic synchrotron background, because the Galactic synchrotron radiation is a foreground contaminator in Cosmic Microwave Background Radiation (CMBR) polarization measurements at high frequencies $v \approx 30-100 \mathrm{GHz}$. Power spectra of the diffuse polarized synchrotron background intensity have been determined from several radio surveys at frequencies from $408 \mathrm{MHz}$ to $2.7 \mathrm{GHz}$, in many parts of the sky (Tucci et al. 2000, 2002; Baccigalupi et al. 2001; Giardino et al. 2002; Bruscoli et al. 2002).

These power spectra studies are based on the following surveys of polarized radiation:

- Dwingeloo 25m-dish survey (Brouw \& Spoelstra 1976), of the region $120^{\circ}<l<180^{\circ}$ and $b>-10^{\circ}$ (although undersampled). This is a multi-frequency survey at $408 \mathrm{MHz}$, $465 \mathrm{MHz}, 610 \mathrm{MHz}, 820 \mathrm{MHz}$ and $1411 \mathrm{MHz}$, with increasing angular resolution of $2.3^{\circ}$ to $0.5^{\circ}$;

- Parkes 2.4 GHz Galactic plane survey (Duncan et al. 1997), of the region $238^{\circ}<l<5^{\circ}$ and with $|b|<5^{\circ}$, at some positions a few degrees higher, at a resolution of $10.4^{\prime}$;
- Effelsberg 2.695 GHz Galactic plane survey (Duncan et al. 1999), of the region $5^{\circ}<l<74^{\circ}$, and $|b|<5^{\circ}$, at a resolution of 4.3';

- Effelsberg 1.4 GHz intermediate latitude survey (Uyanıker et al. 1999), which consists of 4 regions within $45^{\circ}<l<210^{\circ}$ and $-15^{\circ}<b<20^{\circ}$ with an angular resolution of $10.4^{\prime}$;

- Australia Telescope Compact Array (ATCA) 1.4 GHz survey (Gaensler et al. 2001). This is a test region for the Southern Galactic Plane Survey (SGPS, McClure-Griffiths et al. 2001) at $325.5^{\circ}<l<332.5^{\circ},-0.5^{\circ}<b<3.5^{\circ}$, with a resolution of about $1^{\prime}$.

Power spectra of total intensity $I$ and polarized intensity $P$ were derived in these surveys for multipoles over a range of $\ell \approx 10$ to 6000 .

Figure 12 shows the variation of $\alpha_{P}$ with Galactic longitude, latitude and frequency, using the available data as detailed in Table 3. In the left plots, the lines show ranges in longitude (top) and latitude (bottom) over which $\alpha_{P}$ was computed. Solid lines give high multipole numbers $(100<\ell<6000)$, dashed-dotted lines denote an intermediate multipole range $(30<\ell<200)$ and the dotted lines give small multipoles $(10<\ell<80)$. The WSRT data from the Auriga, Horologium en WENSS fields, discussed here, are given in asterisks. In the right plot, $\alpha_{P}$ against frequency is displayed. The connected points are from the same area observed at different frequencies from $408 \mathrm{MHz}$ to $1411 \mathrm{MHz}$ (in the Brouw \& Spoelstra paper).

The first conclusion from Fig. 12 is that multipole spectral indices vary over the sky from $\alpha \approx 1$ to 3 , without showing a clear correlation with Galactic longitude, while only the WENSS subfields show a dependence of spectral index on latitude. However, all surveys have been done at different resolutions and frequencies, and the regions used to compute power 
Table 3. Studies of power spectra of diffuse polarized intensity from different surveys. Given are the range in Galactic longitude $l$ and latitude $b$, the frequency of observation $v$, the range in multipole $\ell$ for which the power spectrum was computed, the spectral index of $P \alpha_{P}$ and the reference to the study of the power spectra.

\begin{tabular}{|c|c|c|c|c|c|c|}
\hline Survey & $l\left(^{\circ}\right)$ & $b\left(^{\circ}\right)$ & $v(\mathrm{MHz})$ & $\ell$ & $\alpha_{P}$ & Reference \\
\hline Dwingeloo & $110-160$ & $0-20$ & 1411 & $30-100$ & 2.9 & Baccigalupi et al. (2001) \\
\hline \multirow{17}{*}{$\begin{array}{l}\text { Brouw \& Spoelstra } \\
\text { (1976) }\end{array}$} & $5-80$ & $50-90$ & ” & $"$ & 3.1 & \\
\hline & $335-360$ & $60-90$ & ” & ” & 2.8 & \\
\hline & $120-180$ & $-10-20$ & 408 & $10-70$ & 1.3 & Bruscoli et al. (2002) \\
\hline & ” & $"$ & 465 & $"$ & 1.1 & \\
\hline & 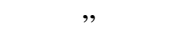 & $"$ & 610 & 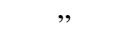 & 1.5 & \\
\hline & ", & $”$ & 820 & ” & 1.5 & \\
\hline & $"$ & $"$ & 1411 & $"$ & 1.8 & \\
\hline & $45-75$ & $26-62$ & 408 & $10-70$ & 1.3 & \\
\hline & $"$ & $"$ & 465 & $"$ & 1.3 & \\
\hline & $"$ & $"$ & 610 & ” & 1.6 & \\
\hline & $"$ & $"$ & 820 & ” & 1.7 & \\
\hline & $”$ & $"$ & 1411 & $"$ & 1.7 & \\
\hline & $15-45$ & $60-84$ & 408 & $10-70$ & 1.1 & \\
\hline & $"$ & $"$ & 465 & $"$ & 0.9 & \\
\hline & 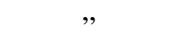 & $”$ & 610 & ” & 1.2 & \\
\hline & ” & $"$ & 820 & $"$ & 1.2 & \\
\hline & $"$ & $"$ & 1411 & $”$ & 1.9 & \\
\hline \multirow{15}{*}{$\begin{array}{l}\text { Parkes } \\
\text { Duncan et al. } \\
\text { (1997) }\end{array}$} & $240-250$ & $-5-5$ & 2400 & $100-800$ & 1.86 & Baccigalupi et al. (2001) \\
\hline & $250-260$ & $"$ & " & $"$ & 1.86 & \\
\hline & $260-270$ & 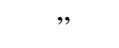 & 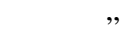 & ” & 1.43 & \\
\hline & $270-280$ & $"$ & $"$ & 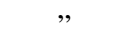 & 1.67 & \\
\hline & $280-290$ & $"$ & $”$ & ” & 1.91 & \\
\hline & $290-300$ & $"$ & " & ” & 1.77 & \\
\hline & $300-310$ & 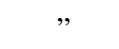 & ” & ” & 1.28 & \\
\hline & $310-320$ & $"$ & $"$ & 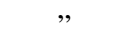 & 1.64 & \\
\hline & $320-330$ & $"$ & $"$ & $"$ & 1.93 & \\
\hline & $330-340$ & $"$ & $"$ & $"$ & 1.44 & \\
\hline & $340-350$ & $"$ & $"$ & ” & 1.47 & \\
\hline & $350-360$ & $"$ & $"$ & 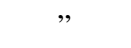 & 1.49 & \\
\hline & $240-360$ & $"$ & $"$ & $"$ & 1.7 & Bruscoli et al. (2002) \\
\hline & $326.5-331.5$ & $-1-4$ & $"$ & $"$ & 1.68 & Tucci et al. (2002) \\
\hline & $240-360$ & $-5-5$ & ” & $40-250$ & 2.4 & Giardino et al. (2002) \\
\hline \multirow{6}{*}{$\begin{array}{l}\text { Effelsberg } \\
\text { Duncan et al. } \\
(1999)\end{array}$} & $20-30$ & $-5-5$ & 2695 & $100-800$ & 1.79 & Baccigalupi et al. (2001) \\
\hline & $30-40$ & $"$ & ” & " & 1.72 & \\
\hline & $40-50$ & $"$ & $"$ & ” & 1.55 & \\
\hline & $50-60$ & $”$ & ” & $”$ & 1.98 & \\
\hline & $55-65$ & $"$ & $"$ & $"$ & 1.93 & \\
\hline & $5-75$ & $"$ & $"$ & $"$ & 1.6 & Bruscoli et al. (2002) \\
\hline \multirow{3}{*}{$\begin{array}{l}\text { Effelsberg } \\
\text { Uyanıker et al. } \\
\text { (1999) }\end{array}$} & $45-55$ & $5-20$ & 1400 & $100-800$ & 1.5 & Bruscoli et al. (2002) \\
\hline & $140-150$ & $4-10$ & $"$ & $"$ & 2.5 & \\
\hline & 190-200 & $8-15$ & ” & $"$ & 2.3 & \\
\hline
\end{tabular}

spectra are of different sizes. This can explain why a possible dependence of $\alpha$ on latitude was not clearly seen in the other studies. The large variation in slopes of angular power spectra in $P$ indicates that interpretation of the slope is not straightforward, possibly due to large influence of depolarization mechanisms. Care must therefore be taken in extrapolating the results to higher frequencies.

Furthermore, spectral indices show an increase with frequency from $408 \mathrm{MHz}$ to $1.4 \mathrm{GHz}$. This means that the power spectra become steeper, so the relative amount of small-scale 
Table 3. continued.

\begin{tabular}{|c|c|c|c|c|c|c|}
\hline Survey & $l\left({ }^{\circ}\right)$ & $b\left(^{\circ}\right)$ & $v(\mathrm{MHz})$ & $\ell$ & $\alpha_{P}$ & Reference \\
\hline ATCA & $327-331$ & $-0.5-3.5$ & 1400 & $600-6000$ & 1.68 & Tucci et al. (2002) \\
\hline Gaensler et al. & $329-332$ & $0-3$ & $"$ & $"$ & 1.66 & \\
\hline \multirow[t]{7}{*}{ (2001) } & $326-329$ & $"$ & $"$ & $"$ & 1.68 & \\
\hline & $330.1-331.1$ & $0.5-1.5$ & $"$ & $"$ & 1.79 & \\
\hline & $329.2-330.2$ & $0.6-1.6$ & $"$ & $"$ & 2.24 & \\
\hline & $327.2-328.2$ & $"$ & $"$ & $"$ & 1.93 & \\
\hline & $328-329$ & $2.1-3.1$ & $"$ & $"$ & 2.58 & \\
\hline & $326.5-327.5$ & $1.8-2.8$ & $"$ & $"$ & 1.88 & \\
\hline & $329.9-330.9$ & $"$ & $"$ & $"$ & 1.68 & \\
\hline WSRT & $158-165$ & $13-20$ & 350 & $100-1000$ & 2.26 & Haverkorn et al. (2003a) \\
\hline WSRT & $134-141$ & $3-10$ & 350 & $100-1000$ & 2.20 & Haverkorn et al. (2003b) \\
\hline \multirow[t]{11}{*}{ WSRT } & $156-143$ & $0.5-7.5$ & 327 & $100-1500$ & 1.67 & Schnitzeler et al., in prep. \\
\hline & $162-169$ & $8-15$ & $"$ & $"$ & 1.48 & \\
\hline & $155.5-161.5$ & $"$ & $"$ & $"$ & 1.84 & \\
\hline & $147.5-154.5$ & $"$ & $"$ & $"$ & 1.70 & \\
\hline & $140.5-147.5$ & $"$ & $"$ & $"$ & 1.63 & \\
\hline & $139-146$ & $15-22$ & $"$ & $"$ & 1.24 & \\
\hline & $146.5-153.5$ & $"$ & $"$ & $"$ & 0.99 & \\
\hline & $153.5-160.5$ & $"$ & $"$ & $"$ & 1.67 & \\
\hline & $153.3-160.5$ & $22-29$ & $"$ & $"$ & 0.73 & \\
\hline & $147-154$ & $"$ & $"$ & $"$ & 0.90 & \\
\hline & $140.5-147.5$ & $"$ & $"$ & $"$ & 0.68 & \\
\hline
\end{tabular}

structure decreases. This could be due to the large Faraday rotation at low frequencies. Typical $R M \mathrm{~s}$ of $5 \mathrm{rad} \mathrm{m}^{-2}$ are present in the Brouw \& Spoelstra data (Spoelstra 1984), and will rotate polarization angles at $325 \mathrm{MHz}$ by about $250^{\circ}$. Variations in $R M$ of a few rad $\mathrm{m}^{-2}$ give angle variations of over $90^{\circ}$, which would cause beam depolarization if the angle variations occur on scales smaller than the beam $\left(2.3^{\circ}\right.$ in this case). Beam depolarization only acts on scales of the synthesized beam, and therefore creates additional structure on small scales in $P$, which flattens the power spectrum. A $\Delta R M$ of $5 \mathrm{rad} \mathrm{m}^{-2}$ would cause a variation in polarization angle of about $40^{\circ}$ at $820 \mathrm{MHz}$, and of no more than $10^{\circ}$ at $1.4 \mathrm{GHz}$. So at frequencies above $1.4 \mathrm{GHz}$, a $\triangle R M$ of $5 \mathrm{rad} \mathrm{m}^{-2}$ would cause negligible beam depolarization. In addition, the resolution of the observations generally increases with increasing frequency, which would also cause a decrease in beam depolarization. This might explain why above $1.4 \mathrm{GHz}$ the spectral index does not appear to be correlated with frequency. The fact that spectral indices in the two WSRT regions are much higher than would be expected from this argument can be due to the criteria used to select the two fields.

\section{Structure functions}

The disadvantage of using angular power spectra is that a regular grid of data is required. If the data are very irregularly spaced (e.g. in the case of data from pulsars or extragalactic point sources), it is better to use the structure function which in principle gives the same information, but can be calculated easily for irregularly spaced data. The structure function $S F$ of a radiation field $X$, as a function of distance lag $d$, is

$S F_{X}(d)=\frac{\sum_{i=1}^{N}\left(X\left(x_{i}\right)-X\left(x_{i}+d\right)\right)^{2}}{N}$

where $X\left(x_{i}\right)$ is the value of field $X$ at position $x_{i}$ and $N$ is the number of data points. If the power spectrum of $R M$ is a power law with spectral index $\alpha$, then the structure function $S F_{R M}$ is

$S F_{R M}(d) \propto d^{\mu}$ where $\mu= \begin{cases}\alpha-2 & \text { for } 2<\alpha<4 \\ 2 & \text { for } \alpha>4\end{cases}$

(Simonetti et al. 1984). We determined the structure functions of $R M$ to compare with existing estimates of the structure function of Galactic $R M$ from polarized extragalactic point sources. As the determination of structure functions does not require a regular grid, we can compute the $S F$ including and excluding "bad" data points to examine how the structure functions of $P$ and $R M$ change. This will allow us to estimate the effect of "bad" data in the power spectra in $P$ and $R M$ computed in Sect. 3.

\subsection{Structure functions of $R M$}

Structure functions $S F_{R M}$ in the Auriga and Horologium regions are plotted against distance $d$ in degrees in the log-log 

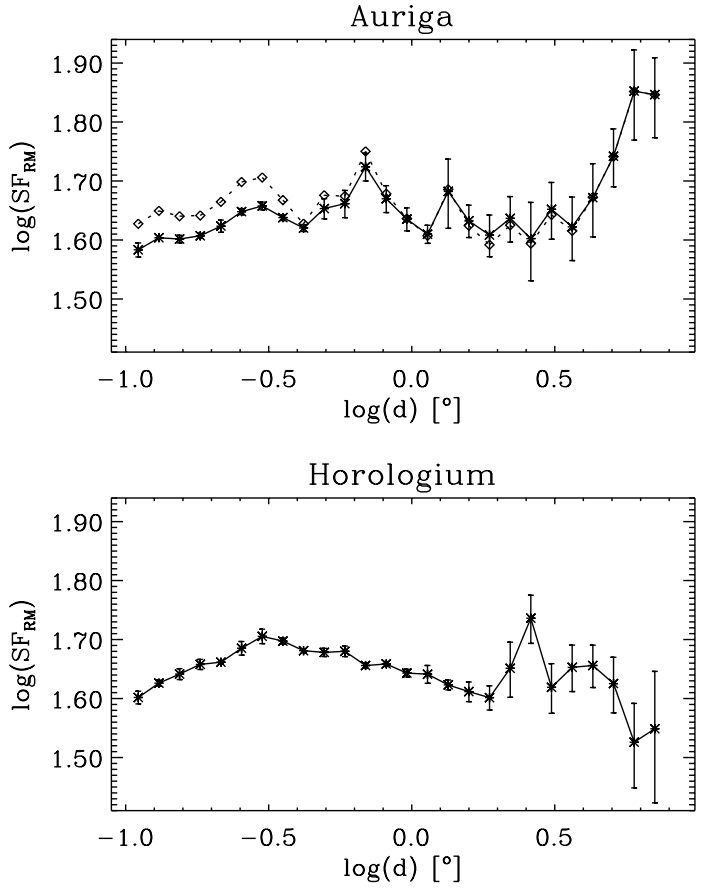

Fig. 13. The solid lines show the $R M$ structure function $S F_{R M}$ as a function of distance $d$ in degrees for the Auriga region (top) and the Horologium region (bottom), using only reliable $R M$ values. The dotted line in the top plot gives $S F_{R M}$ evaluated for the entire grid, including unreliable $R M$ determinations.

plots in Fig. 13 (solid line), where error bars denote the standard deviation. The minimum distance shown is $d \approx 7^{\prime}$. For the evaluation of the $S F$, only "reliably determined" $R M$ values are used, according to the definition in Sect. 2.1. Although the spectrum is consistent with a flat slope, there is some evidence for a break in the slope at $d=0.3^{\circ}$, primarily in the Horologium field. For larger angular scales, the $S F$ is approximately flat in the Auriga region, with a tentative increase at the largest lags, and even decreasing in Horologium.

We can estimate the magnitude of the contribution of unreliably determined $R M$ s by reevaluating the $S F$ for the complete grid of $R M$ values, instead of only the reliable $R M \mathrm{~s}$. This estimate is important for a discussion of the power spectra of $R M$, which were evaluated over the complete dataset, including unreliably determined $R M \mathrm{~s}$. The structure function using the complete dataset is shown in the left panel of Fig. 13 as a dotted line. The structure function clearly has a lower amplitude if the unreliable $R M$ determinations are removed, but the slope of the structure function remains approximately the same.

\subsection{Structure functions of $P$}

We compute the structure function of polarized intensity, for both the complete grid of beams, and for those beams selected to have high $P$. In Fig. 14 we show structure functions of $P$ in the Auriga region for 5 frequencies, where again the error bars denote the standard deviation. The average logarithmic slope of the structure functions is $\sim 0.35$ in the range $0.2^{\circ} \lesssim d \lesssim 1^{\circ}$, and the spectrum flattens on larger scales, probably due to the insensitivity of the WSRT to structure on large angular scales. The solid line denotes the estimate based only on beams with $P>4 \sigma$, and the dotted line gives the estimate based on all the data. The structure functions based on selected beams and those based on all data are not significantly different on small scales, and start deviating only for $d \gtrsim 0.7^{\circ}$. Although small deviations are created by selecting the best data, the overall slope of the structure function is hardly affected by the selection including only regions of high $P$. The relatively small effect of including bad data gives confidence that the determination of the logarithmic slope of the power spectra of $R M$ and $P$ is not significantly influenced by noisy data or poorly determined $R M$.

\section{Discussion}

To the authors knowledge, the results presented here are the first observational determinations of the power spectrum of rotation measure. What power spectrum is to be expected is unclear, as the $R M$ is a complex quantity depending on magnetic field structure and direction, electron density and the path length through the ionized ISM. There are indications that the electron density and $R M$ exhibit Kolmogorov turbulence (Minter \& Spangler 1996; Armstrong et al. 1995).

An approximately flat structure function corresponds to structure of equal amplitude on all scales, similar to a noise spectrum. The break in $S F_{R M}$ at $\delta \theta \approx 0.3^{\circ}$, if present, corresponds to a change in characteristics of the structure on scales of $\sim 3.9 \mathrm{pc}$, assuming a path length of $600 \mathrm{pc}$ (Haverkorn et al. 2003d).

Minter \& Spangler (1996) studied structure functions of $R M$ from polarized extragalactic sources and of emission measure $(E M)$ from $\mathrm{H} \alpha$ measurements, on the same angular scales as in our observations. They find a break in the slope of the structure function, which can be interpreted as a transition of 3D Kolmogorov turbulence $(\mu=5 / 3$, where $\mu$ is the slope of the structure function as defined in Eq. (3)) to $2 \mathrm{D}$ turbulence $(\mu=2 / 3)$ in $R M$ on angular scales of $\delta \theta \approx 0.1^{\circ}$, the scale of our resolution. They assume a total path length of $2000 \mathrm{pc}$, and conclude that the transition occurs at scales of $\sim 3.6 \mathrm{pc}$, consistent with the spatial scale at which we estimate the turnover point in the $S F$ of the Auriga and Horologium regions. However, there is a large discrepancy between $\mu$ values found by Minter \& Spangler $(\mu=5 / 3$ and $2 / 3)$ and by us $(\mu \approx 0)$, which could be explained by the fact that they probe the complete line of sight through the medium of many kpc, whereas the $R M$ that we obtain is only produced in the nearest $\sim 600 \mathrm{pc}$. Therefore, it is possible that the nature of the turbulence changes from $\mu=0$ nearby (approximately in the Galactic stellar disk), to $2 \mathrm{D}$ and/or Kolmogorov-like turbulence at larger distances.

However, flat structure functions are also found by Simonetti et al. (1984), who have studied structure functions from $R M \mathrm{~s}$ in three regions of the sky. Two of the three regions (around the North Galactic Pole and at $180^{\circ}<l<220^{\circ}$, $10^{\circ}<b<50^{\circ}$ ) are consistent with a flat structure function $(\mu=0)$. (The third region is located along the local arm and in the plane, at $70^{\circ}<l<110^{\circ}$ and $-45^{\circ}<b<5$, and its structure function of $R M$ has a slope $\mu \approx 1$.) The smallest angular scale they probe in these regions is about $2^{\circ}$. Clegg et al. (1992) study 


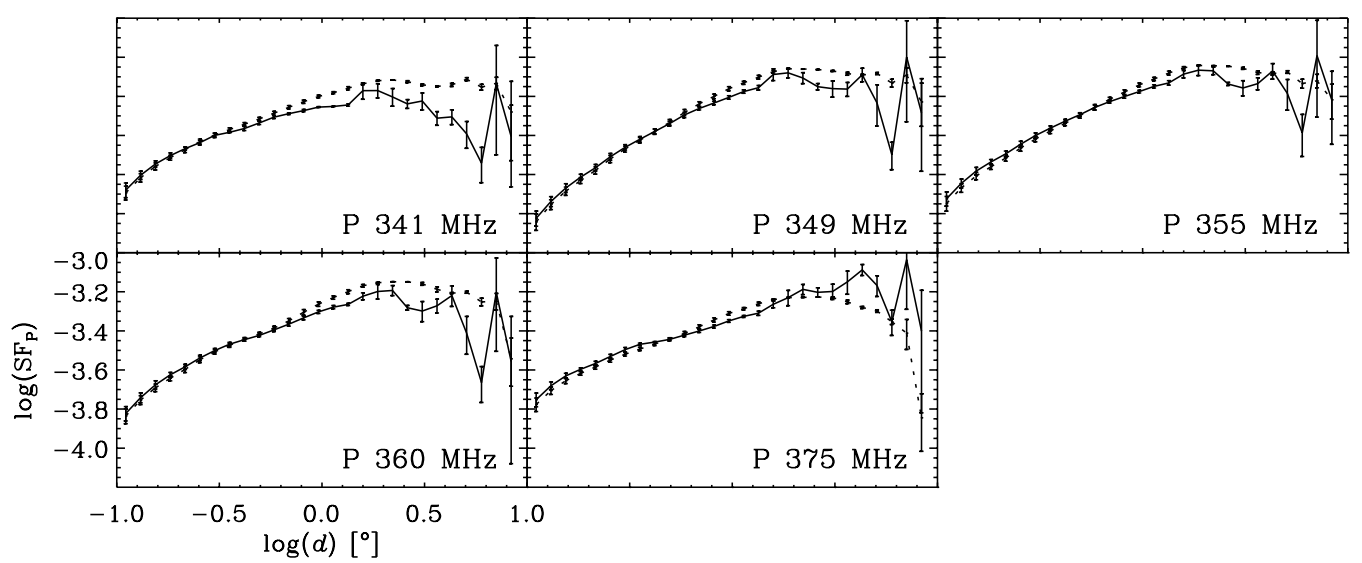

Fig. 14. Structure function $S F_{P}$ as a function of distance $d$ in degrees for the Auriga region, in 5 frequencies. The solid line shows $S F_{P}$ in which only beams with $P>4 \sigma$ are included, the dotted line gives $S F_{P}$ as computed from all data.

low-latitude extragalactic sources and also obtain flat structure functions, albeit with a much higher amplitude than that of the high-latitude extragalactic sources.

\section{Conclusions}

The multipole spectral index for polarized intensity $P$ is $\alpha \approx 2.2$ in the Auriga and Horologium regions, and ranges from 0.7 to 1.8 for subfields in the WENSS polarization region. The multipole spectral index decreases with Galactic latitude (i.e. power spectra become flatter towards higher latitudes), but is probably constant with Galactic longitude.

In all regions, the power spectra of Stokes $Q$ and $U$ are steeper than the spectra of $P$. This is most likely due to the presence of a Faraday screen, which creates additional structure in $Q$ and $U$, but not in $P$. As the Faraday screen is located in front of the polarized emission, the structure induced by the screen will be on larger angular scales than that of the emission, which steepens the $Q$ and $U$ spectra. The derived power spectra of $P$ agree with earlier estimates, although all estimates show a large range of $0.6 \lessgtr \alpha_{P} \lesssim 3$, possibly due to a large influence of depolarization mechanisms. This makes interpretation of the power spectra uncertain.

Structure functions of $R M$ in the Auriga and Horologium fields are consistent with flat spectra (i.e. a logarithmic slope of the structure function $\mu=0$ ), but may show a break close to $0.3^{\circ}$, which is at the same spatial scales as a break in the structure function in the $R M \mathrm{~s}$ of extragalactic sources (Minter $\&$ Spangler 1996). The flat spectrum indicates a noise-like spectrum with equal amounts of structure on all scales.

The derived structure functions support the estimates of the power spectra, because they are based only on high-quality data, i.e. reliably determined $R M$, and $P$ with high $S / N$. Structure functions using all data do not show a significant difference with the structure functions of the selected data. This gives confidence that the power spectra determinations are not significantly affected by bad data points.

Acknowledgements. We thank B. J. Rickett for helpful discussions and suggestions, and F. Heitsch for help in constructing and analyzing power spectra. The Westerbork Synthesis Radio Telescope is operated by the Netherlands Foundation for Research in Astronomy (ASTRON) with financial support from the Netherlands Organization for Scientific Research (NWO). Computations presented here were performed on the SGI Origin 2000 machine of the Rechenzentrum Garching of the Max-Planck-Gesellschaft. MH acknowledges the support from NWO grant 614-21-006.

\section{References}

Armstrong, J. W., Rickett, B. J., \& Spangler, S. R. 1995, ApJ, 443, 209

Baccigalupi, C., Burigana, C., Perrotta, F., et al. 2001, A\&A, 372, 8

Brouw, W. N., \& Spoelstra, T. A. T. 1976, A\&AS, 26, 129

Bruscoli, M., Tucci, M., Natale, V., et al. 2002, NewA, 7, 171

Chevalier, R., \& Fransson, C. 1984, ApJ, 279, L43

Clegg, A. W., Cordes, J. M., Simonetti, J. H., \& Kulkarni, S. R. 1992, ApJ, 386, 143

Duncan, A. R., Reich, P., Reich, W., \& Fürst, E. 1999, A\&A, 350, 447

Duncan, A. R., Haynes, R. F., Jones, K. L., \& Stewart, R. T. 1997, MNRAS, 291, 279

Ferrière, K. M. 2001, RvMP, 73, 1031

Gaensler, B. M., Dickey, J. M., McClure-Griffiths, N. M., et al. 2001, ApJ, 549, 959

Giardino, G., Banday, A. J., Górski, K. M., et al. 2002, A\&A, 387, 82 Gray, A. D., Landecker, T. L., Dewdney, P. E., \& Taylor, A. R. 1998, Nature, 393, 660

Han, J. L., Manchester, R. N., \& Qiao, G. J. 1999, MNRAS, 306, 317

Haslam, C. G. T., Stoffel, H., Salter, C. J., \& Wilson, W. E. 1982, A\&AS, 47, 1

Haslam, C. G. T., Klein, U., Salter, C. J., et al. 1981, A\&A, 100, 209

Haverkorn, M. 2002, Ph.D. Thesis, Leiden Observatory

Haverkorn, M., Katgert, P., \& de Bruyn, A. G. 2003a, A\&A, in press [astro-ph/0303575]

Haverkorn, M., Katgert, P., \& de Bruyn, A. G. 2003b, A\&A, in press [astro-ph/0304087]

Haverkorn, M., Katgert, P., \& de Bruyn, A. G. 2003c, A\&A, submitted Haverkorn, M., Katgert, P., \& de Bruyn, A. G. 2003d, A\&A, submitted Haverkorn, M., Katgert, P., \& de Bruyn, A. G. 2000, A\&A, 356, L13 Katgert, P., \& de Bruyn, A. G. 1999, in New perspectives on the interstellar medium, ed. A. R. Taylor, T. L. Landecker, \& G. Joncas, p. 411

Landecker, T. L., Uyanıker, B., \& Kothes, R. 2001, AAS, 199, \#58.07

McClure-Griffiths, N. M., Green, A. J., Dickey, J. M., et al. 2001, ApJ, 551,394 
Minter, A. H., \& Spangler, S. R. 1996, ApJ, 458, 194

Minter, A. H., \& Balser, D. 1997, ApJ, 484, L133

Ohno, H., \& Shibata, S. 1993, MNRAS, 262, 953

Ostriker, E. C., Stone, J. M., \& Gammie, C. F. 2001, ApJ, 546, 980

Prunet, S., Sethi, S. K., \& Bouchet, F. R. 2000, MNRAS, 314, 348

Rand, R. J., \& Kulkarni, S. R. 1989, ApJ, 343, 760

Rand, R. J., \& Lyne, A. G. 1994, MNRAS, 68, 497

Rengelink, R. B., Tang, Y., de Bruyn, A. G., et al. 1997, A\&AS, 124, 259

Seljak, U. 1997, ApJ, 482, 6

Simard-Normandin, M., \& Kronberg, P. P. 1980, ApJ, 242, 74

Simonetti, J. H., \& Cordes, J. M. 1986, ApJ, 303, 659
Simonetti, J. H., Cordes, J. M., \& Spangler, S. R. 1984, ApJ, 284, 126 Spoelstra, T. A. T. 1984, A\&A, 135, 238

Tucci, M., Carretti, E., Cecchini, S., et al. 2002 [astro-ph/0207237]

Tucci, M., Carretti, E., Cecchini, S., et al. 2000, NewA, 5, 181

Uyanıker, B., Fürst, E., Reich, W., Reich, P., \& Wielebinski, R. 1999, A\&AS, 138, 31

Uyanıker, B., \& Landecker, T. L. 2002, ApJ, 575, 225

Vázquez-Semadeni, E., \& Passot, T. 1999, in Interstellar Turbulence, ed. J. Franco, \& A. Carramiñana, p. 223

Wieringa, M. H., de Bruyn, A. G., Jansen, D., Brouw, W. N., \& Katgert, P. 1993, A\&A, 268, 215 